\title{
Artificial fruit for monitoring the thermal history of horticultural produce in the cold chain
}

Thijs Defraeye ${ }^{a, b} *$, Wentao $\mathrm{Wu}^{b}$, Kevin Prawiranto ${ }^{\mathrm{a}, \mathrm{b}}$, Giuseppino Fortunato ${ }^{c}$, Shelley Kemp ${ }^{c}$, Stefan Hartmann ${ }^{d}$, Paul Cronje ${ }^{e}$, Pieter Verboven ${ }^{f}$, Bart Nicolai ${ }^{f, g}$

${ }^{a}$ Multiscale Studies in Building Physics, Empa, Überlandstrasse 129, 8600 Dübendorf, Switzerland

${ }^{b}$ Chair of Building Physics, ETH Zurich, Stefano-Franscini-Platz 5, 8093 Zürich, Switzerland

${ }^{c}$ Laboratory for Protection and Physiology, Empa, Lerchenfeldstrasse 5, 9014 St. Gallen, Switzerland

${ }^{d}$ Center for X-ray Analytics, Empa, Überlandstrasse 129, 8600 Dübendorf, Switzerland

${ }^{e}$ Citrus Research International, Department of Horticultural Sciences, Stellenbosch University, Stellenbosch 7602, South Africa

${ }^{f}$ KU Leuven - University of Leuven, Division MeBioS, Postharvest Group, Willem de Croylaan 42, 3001 Heverlee, Belgium

${ }^{g}$ VCBT, Flanders Centre of Postharvest Technology, Willem de Croylaan 42, 3001 Heverlee, Belgium

\section{* Corresponding author}

- E-mail thijs.defraeye@empa.ch

- Tel. $\quad+41(0) 587654790$

- $\quad$ Fax. $\quad+41446331041$

Keywords: fruit simulator, convective cooling, apple, sensor, temperature, biomimetic

This document is the accepted manuscript version of the following article:

Defraeye, T., Wu, W., Prawiranto, K., Fortunato, G., Kemp, S., Hartmann, S., ... Nicolai, B. (2017).

Artificial fruit for monitoring the thermal history of horticultural produce in the cold chain. Journal of Food Engineering, 215, 51-60. http://doi.org/10.1016/j.jfoodeng.2017.07.012

This manuscript version is made available under the CC-BY-NC-ND 4.0

license http://creativecommons.org/licenses/by-nc-nd/4.0/ 


\section{Abstract}

There is a need for more realistic monitoring of fruit pulp temperature history throughout the cold chain at a higher spatial resolution inside the cargo. Particularly solutions that are easy to install in a commercial setting are required. For this purpose, a novel kind of fruit simulator - an artificial fruit - has been designed, manufactured and tested. Using a biomimetic approach, it was engineered specifically to match the thermal response of real fruit as close as possible. The artificial fruit is composed out of a thin plastic shell, which mimics the exterior size, shape, surface texture and color of the fruit of interest. This shell is filled with a mixture that has similar thermal properties as real fruit as it is basically composed out of the same components. Two self-powered data loggers with a built-in sensor are integrated in the artificial fruit. These different components of the artificial fruit were combined successfully into a manufactured prototype. The thermal response of an artificial apple fruit during cooling was evaluated against that of 10 real apples. The cooling time of the artificial fruit was within $5 \%$ of that of the real fruits. The artificial fruit also had a more accurate thermal response than that of water-filled fruit simulators, with which differences up to $16 \%$ were found. The uniformity in size, shape and sensor location of the artificial fruit also enables it to measure fruit pulp temperature in a much more repeatable manner than with real fruit. A particular advantage of the artificial fruit is that it can be packed directly with the fresh produce in a commercial setting, by which multiple locations inside the cargo can easily be monitored. This novel sensor system thus provides an improved method to identify heterogeneities in cargo cooling, and associated quality issues. The artificial fruit is especially of interest for monitoring fruit pulp in precooling facilities, cold stores, ripening facilities and refrigerated containers. 


\section{Introduction}

\subsection{Background}

Postharvest losses in fresh produce cold chains, from the point of harvest until the product reaches the consumer vary between $13 \%$ (Europe) and 38 \% (sub-Saharan Africa) (Gustavsson et al., 2011). Here, temperature is the single most important parameter affecting produce quality deterioration, ripening rate and shelf life. To maintain fruit quality and to reduce these losses, rapid removal of the field heat after harvest through cooling is thus of key importance, as well as maintaining optimum product temperature throughout the postharvest supply chain. These cold-chain unit operations include forced-air precooling, long-haul maritime transport in refrigerated containers, transport in refrigerated trucks as well as long-term storage in cold rooms prior to distribution to the end client.

Monitoring postharvest fruit temperature history of fresh fruits is thus essential to evaluate the efficacy of existing commercial cold-chain unit operations but also to explore new cooling strategies. Typical examples of such recent cooling strategies are flow reversal for forced-air precoolers (Ferrua and Singh, 2009), ambient loading for refrigerated containers (Defraeye et al., 2015b) and intermittent operation of cooling fans in cold storage rooms and refrigerated containers (Ambaw et al., 2016; Lukasse et al., 2011; Thompson, 2004). Fruit temperature measurements are also essential to evaluate and develop new ventilated bulk packaging (Gruyters et al., 2017; O'Sullivan et al., 2016; Wu et al., 2017). Here, the fruit cooling rate is a major design criterion (Defraeye et al., 2015a, 2013), next to mechanical strength (Berry et al., 2017). Fruit temperatures are also indicators of problematic hot spots within the cargo during shipment of commodities with a high respiration rate, such as bananas. Such hot spots can induce spontaneous ripening of the cargo during transport and should be avoided (Jedermann et al., 2014c). In commercial fresh produce export, the fruit core or pulp temperature measurement is used by governmental organizations such as the U.S. Department of Agriculture - Animal and Plant Health Inspection Service (USDA-APHIS) and the South-African parastatal organization Perishable Products Export Control Board (PPECB). These organizations use this data to determine the compliance of the cargo with cold treatment protocols designed to kill specific phytosanitary organisms (e.g. fruit fly, false codling moth). The core of these protocols is that the fruit core temperature must be maintained below a certain temperature threshold for a specific duration. These cold treatment protocols can be applied as land-based treatments but most often are done in-transit from the export country to the target market in refrigerated containers (USDA-

APHIS, 2017). Customers (wholesalers) seem also willing to pay higher prices and to purchase larger quantities of fruit when the quality of the cargo is accurately monitored (Choe et al., 2009; Pang et al., 2015). Even insurance companies encourage their clients to monitor the thermal history of their cargo by giving them a discount (Pang et al., 2015; Swedberg, 2011).

\subsection{Sensor technology for fruit temperatures}

Several sensors/logger systems are currently used to monitor fruit temperature. Wired sensors, such as point probes (e.g. thermocouples), are placed inside the fruit core to measure pulp temperature (Defraeye et al., 2013). These measurements can be done continuously throughout the entire unit operation, for example during transit, or at a certain point in time, for example upon arrival of the cargo. These sensors are standard equipment in 
refrigerated containers and precoolers. Wireless, self-powered data loggers with a built-in sensor, such as iButtons $^{\circledR}$, have also been used to measure core temperatures of fruit (Defraeye et al., 2016, 2015b; Hoang et al., 2012). They are typically placed on the fruit surface or inside the fruit core by making an incision.

Smart sensor tags, often based on radio frequency identification (RFID), have been used to measure air temperatures inside the cargo. Some of these devices also included monitoring of metabolic gasses, but such systems are still in a development stage ((EU-Catrene, 2012; Jimenez-Ariza et al., 2014; Laniel et al., 2011; Laniel and Émond, 2010; Pang et al., 2015; Zou et al., 2014)). Depending on the type of device (i.e. active, passive, battery-assisted passive), they log the data on the tag for later wireless readout, or directly transmit the data wireless to an external data logger base station in the vicinity of the tag. Due to the advantage of wireless readout, the main idea behind these sensor tags is that they can be placed on each carton/box of the fresh cargo or at least on each pallet. Both disposable and reusable RFID-based sensors are commercially available from several suppliers, as well as temperature loggers based on Bluetooth technology. There are however several limitations to the current temperature measurements of fruit, particularly for commercial cold-chain operations.

\subsection{Current restrictions of temperature measurements}

A first problem is that the air or fruit surface temperature is often monitored in commercial operations, instead of the fruit (pulp) temperature (Defraeye et al., 2015a; Jedermann et al., 2014a). A typical example is RFID sensor tags, which are used to log the cold chain's thermal history. Air or fruit-surface temperatures pick up instantaneous changes in ambient cooling conditions, whereas fruit core temperatures lag behind due to the thermal inertia of the fruit. As such, the fruit does not react that fast in terms of its temperature on fluctuations in air temperature due to intermittent cooling/ventilation. The fruit core is usually the last location to attain the set temperature so is the most conservative location to measure temperature. Pulp temperatures reflect better the thermal condition of the fruit, and are a better measure for the evolution of the fruit quality and thus shelf life. This is why pulp temperatures - not air temperatures - are measured in several commercial unit operations in the postharvest cold chain. It is used by governmental organizations to decide upon compliance with any phytosanitary cold protocol during overseas or land-based transport, as well as in commercial forced-air precooling (PPECB, 2016a, 2016b; Thompson, 2008; USDA-APHIS, 2017) to asses if the cooling process can be stopped.

A second problem is that typically a limited amount of sensors/loggers is installed inside the cargo in commercial operations, for example 3 PT-100 point probes (connected to the reefer) per 20 pallets in a 40 feet container (Defraeye et al., 2016), or 3-5 point probes per 40 pallets in a precooler (Wu et al., 2017). As such, the heterogeneity of fruit cooling rates for different cartons in the cargo is not correctly picked up and will lead to inhomogeneous fruit quality or possibly non-compliance with a cold treatment protocol. Such heterogeneity has been identified (Defraeye et al., 2016; Dehghannya et al., 2012; Delele et al., 2013a, 2013b; do Nascimento Nunes et al., 2014; Olatunji et al., 2017) and is present at various scales: inside a carton of fruit, between stacked cartons on a pallet as well as between different pallets/palloxes in a container cargo or a storage room. RFID sensor tags could mitigate this problem of picking up the heterogeneity, as they are typically placed at multiple locations. However, these tags do not measure pulp temperature. 
A third problem is that sensors to measure fruit pulp temperature are often installed at easy accessible places and do not measure deep inside a pallet or carton. These are, however, the critical locations in terms of cooling rates or high ethylene levels. These issues are caused by the fact that installing and retrieving the sensors inside different pallets is cumbersome and labor/time intensive. For wired sensors, for example, the wiring is intrusive, requires a lot of cabling and typically a connection to an external data logger for continuous measurements. RFID tags, on the other hand, cope with strong signal attenuation, especially within fruit bulks which are densely packed, by which message forwarding can be necessary in a refrigerated container (Jedermann et al., 2014b). Fourth, measurements tracking the fruit pulp thermal history throughout its entire cold chain, from farm to retailer, are rare, particularly for long (overseas) chains. One reason is the numerous and different stakeholders owning and operating the various equipment, who are sequentially responsible for the cargo during subsequent unit operations. This makes the use of a generic pulp temperature recording platform problematic. The use of wired sensors, transferring between unit operations is hindered by the cabling and the data logger. The small wireless loggers, which are inserted into the fruit core, also cause problems as this intrusive practice wounds the fruit. Such wounds induce enzymatic reactions and microbial activity, leading to fruit decay and cross contamination. RFID sensor tags have been designed to track the fruit throughout its entire cold chain journey, in order to make in-transit decisions on cold chain logistics (East, 2011; Hertog et al., 2014; Jedermann et al., 2014a; Lütjen et al., 2013). Nevertheless, the ability to measure air and not fruit pulp temperatures at the package or pallet surface restrict their use for monitoring in some cold chain applications.

Academic studies have already addressed several of these aspects, for example by installing more sensors in a cargo (Defraeye et al., 2015b; Hoang et al., 2012; Jedermann et al., 2013; Wu et al., 2017) or by measuring throughout an entire chain (Defraeye et al., 2016; do Nascimento Nunes et al., 2014). Nevertheless, it is clear that installing similar test setups on a commercial level is too time-consuming and also requires skilled personnel, including for data processing and interpretation. We clearly need a solution for more realistic monitoring of the postharvest pulp temperature history of fresh fruits throughout the entire chain at a higher spatial resolution in the cargo, which is easy to install in a commercial setting.

\subsection{Study scope}

This study aims to accommodate this need by developing an artificial fruit. A biomimetic approach is applied to create such a fruit simulator that looks and reacts thermally similar to the real fruit species of interest. This artificial fruit is instrumented with self-powered data loggers with a built-in temperature sensor to monitor core (and surface) temperatures. The main advantages of this novel sensor system are that:

- It is a realistic way of monitoring the fruit's thermal behavior throughout the cold chain, as it mimics measuring the pulp temperature inside a real fruit.

- The artificial fruit can be packed directly with the fresh produce in the designated packaging in a commercial setting. Multiple locations can thus be monitored inside the cargo, also those very deep inside a pallet for example, and such monitoring can be done throughout the entire cold chain.

- The artificial fruit is a stand-alone unit (no external cabling, loggers or other hardware) by which it does not affect the airflow field and cooling behavior of surrounding produce. This enables easy installation 
during packaging and palletization with no additional handling throughout subsequent cold chain operations until sensor retrieval, for example at the retailer.

- The artificial fruits can be reused many times over their service life (estimated 4-7 years) and do not spoil, in contrast to real fruit.

This study proposes the concept of this special type of fruit simulator and its components (section 2), and evaluates its thermal performance by comparing with real fruits (section 3). 


\section{Materials and methods}

\subsection{Produce simulator materials}

Produce simulators have been used to measure fruit cooling by monitoring the core temperature (Allais et al., 2006; Alvarez et al., 2003; Larissa R. De Castro et al., 2005; Dehghannya et al., 2012; Delele et al., 2013b; Lu et al., 2009; Vigneault et al., 2006; Vigneault and de Castro, 2005) (Table 1), predominantly in academic studies rather than commercial shipments. Inside the core of these (predominantly) spherical simulators, a thermocouple was inserted which was connected to an external data logger. Produce simulators do not exhibit (biological) variability within individual products and increase experimental repeatability, compared to real fruit. Smooth solid spheres made out of a polymer (e.g. snooker ball, (Alvarez et al., 2003; Larissa R. De Castro et al., 2005; Dehghannya et al., 2012; Vigneault et al., 2006; Vigneault and de Castro, 2005)) or aluminum (Lu et al., 2009) were used (Defraeye et al., 2015a). However, they differ significantly in terms of thermal properties compared to a real fruit. If no appropriate scaling is made, the predicted cooling behavior will be significantly different. Simulators filled with a water-based solution (Allais et al., 2006; De Castro et al., 2004; Delele et al., 2013b) have also been used, which have thermal properties that are closer to real fruit.

Real fruit are typically composed of $80 \%$ water and around $20 \%$ carbohydrates (in weight), but also have a significant portion of air in the intercellular spaces ( $20 \%$ in volume for apple). This leads to a different density, thermal capacity and thermal conductivity than normal water. The presence of carbohydrates also lowers the freezing point. Furthermore, the water is immobile at the macroscopic level in real fruit. In water-filled spheres however, mixing can occur due to shaking during transport or natural convective flow due to temperature gradients during cooling. This can alter the internal heat transfer rate as a result. Therefore, some researchers immobilized the water by using a gelling agent (De Castro et al., 2004; L. R. De Castro et al., 2005). The food simulant Tylose, a cellulose-based material, is another option (Chuntranuluck et al., 1998), and contains about 75 $\%$ water. Furthermore, only smooth spherical produce simulators have been used, which do not reflect the actual shape or surface texture of many horticultural produce. In general, little research targeted the design of these simulators, as confirmed by a recent review on the topic (Redding et al., 2016).

The previously-used fruit simulators originated from finding a straightforward alternative for real fruit, rather than from engineering a simulator that reacts exactly the same as a real fruit and that could be easily applied in a commercial setting. The artificial fruit presented here, on the other hand, is engineered using a thermobiomimetic approach. The shape, composition and thermal properties of the artificial fruit are carefully tuned to match those of the fruit of interest, by which it reacts similar to a real fruit, and without any external cabling being present. Thereby, it can be used in a straightforward way in commercial facilities.

\subsection{Artificial fruit components}

The artificial fruit should react thermally similar (conduction, convection, radiation) as the fruit species of interest in order to provide realistic temperature data. To this end, the artificial fruit is composed out of a thin plastic shell, which mimics the exterior size, shape, surface texture and color of the fruit of interest (Figure 1). This hollow shell is filled with a water-carbohydrate-air gel mixture (WCA), based on the actual internal fruit composition, to have similar thermal properties as real fruit (conductivity, density, heat capacity, freezing point). 
Two self-powered data loggers with a built-in sensor were integrated in the artificial fruit: one to measure the fruit core temperature and one to measure the temperature near the surface. These components are discussed in detail below. The concept of the fruit simulator is illustrated in this study for apple fruit (Malus $x$ domestica Borkh.), specifically for the cultivar Braeburn, but can easily be applied to other fruit species or cultivars. In contrast to previous fruit simulators (Table 1), all components of this artificial fruit were specifically engineered to match the thermal behavior of the fruit as close as possible.

\subsection{Artificial fruit shell}

\subsubsection{Fruit shape information}

A geometrical model generator for horticultural products was used to generate a three-dimensional CAD model of the artificial fruit, of which the details are published previously (Rogge et al., 2015, 2014). As a first step, nondestructive imaging (X-ray computed tomography) was used to obtain the three-dimensional size and shape of the outer surface of the target fruit. In this study, 73 X-ray scans of Braeburn apple were made (Rogge et al., 2014). Note that also interior composition details can also be inferred from such imaging. Examples are the size and shape of the stone for mango fruit, or the thickness of the rind for orange fruit.

Edge detection was used to extract the 3D surface contour of the outer surface for each fruit from the scans. With this digital shape information of a batch of fruit, a statistical shape description based on Fourier descriptors was performed. As a result, the average outer surface contour of the fruit was determined. Both the 2D average contour (for axisymmetric fruit (Rogge et al., 2014)), and directly a 3D model of the entire fruit (Rogge et al., 2015) were available. In the present study, we used the 2D average contour and obtained the 3D fruit shape by revolution around the symmetry axis. The average total volume of the apple fruit was $0.20 \mathrm{~L}$. From this geometrical information, a 3D CAD model (.STL, .IGES) was generated, which was used as input for the design of the shell of the artificial fruit. This model represents the average shape (and size) of a Braeburn apple fruit.

\subsubsection{Shell model}

The shell of the fruit simulator has several functions. First, it should mimic the shape, size and, if necessary also the surface texture of real fruit, and have a similar thermal behavior. To this end, the average shape of the apple fruit was used for the shell's outer contour, the shell was made out of a material which has thermal properties close to those of real apple fruit (see below, Table 2), and the shell was given a small thickness ( $2 \mathrm{~mm}$ ). Second, the shell should serve as a container for the water-carbohydrate-air gel filling. To this end, the shell was made hollow and watertight, with a hole for filling. Third, easy access to the sensor/logger (iButton ${ }^{\circledR}$ ) in the core of the fruit should be possible. For this purpose, the artificial fruit was split up in two hollow parts (Figure 1, hemispheres), with an enclosure for embedding the temperature loggers. By means of small magnetic inlays, the two halves can be easily disassembled.

The outer shell was designed in a CAD software and is manufactured via additive manufacturing. Selective laser sintering with polyamide (PA2200, Table 2) was used (Materialise NV, Belgium), which was approved as 'food- 
safe' by FDA. The polyamide models were sealed with a low-viscocity copolymer adhesive sealant (Captain Tolley's, UK) to make them watertight. Afterwards, the shell was painted red with a spray paint based on a synthetic resin.

Additive manufacturing is chosen since only a small numbers of artificial fruit needed to be produced. This technology is most suitable if small batches of models need to be made, for example cultivar- or species-specific models, requiring an customized fruit shape. If large amounts need to be produced, other techniques such as injection molding would be more suitable. The advantage of additive manufacturing is that no additional assembly of components is required afterwards. In addition, different compartments can be incorporated in the shell if a fruit has zones with different thermal properties (e.g. large pit in mango, air space within bell pepper).

\subsubsection{Filling}

Fruit is composed predominantly out of water and carbohydrates, with a mass fraction of around $85 \%$ and $15 \%$, respectively for apple fruit, and additionally also little amounts of fat and proteins (ASHRAE, 2010). The internal intercellular air space, so porosity, occupies about 10-25\% in volume (Herremans et al., 2015, 2013), depending on the cultivar but also harvest year and location. The composition of several fruits can be found in tables (ASHRAE, 2010). The porosity can be found in literature (Herremans et al., 2015), or can be measured. The latter can be revealed by X-ray imaging measurements (Herremans et al., 2015, 2013). However, a more simple technique can also be used, namely by determining the mass and volume of fruit samples, and calculating the porosity based on a known fruit tissue density. For the Braeburn apple used in this study, the porosity was determined that way and was found to be $13 \%$ on average. To obtain a thermal performance which is as close as possible to real fruit, a biomimetic approach is used to design the filling. To this end, the filling is composed out of the three basic components of fruits: water, carbohydrates and air, which are added in the correct relative amount for the target fruit species (Table 2). The thermal properties, calculated for apple fruit according to (ASHRAE, 2010), agreed well with other reported values (Martínez-Monzó et al., 2000; Redding et al., 2016).

A water-soluble carbohydrate was used, i.e. a disaccharide, namely sucrose. Since carbohydrates were added to the water, the freezing point drops below $0^{\circ} \mathrm{C}$, as with real fruit. To mimic the air in the intercellular spaces in fruit, highly porous microspheres were used, namely expanded polystyrene (EPS) particles with closed internal pores (diameter 0.5-1.5 mm).

A gelling agent (i.e. polysaccharide agar, commercially available) was added in a low concentration to immobilize the liquid mixture and to make sure the light microspheres maintained an even distribution in the gel. These elastic, thermoreversible gels have a gelling (setting) temperature around $35-70^{\circ} \mathrm{C}$ and a melting temperature around $80-90^{\circ} \mathrm{C}$, which are strongly dependent on the concentration in which the gelling agent is applied. A mass fraction below one percent was found to be sufficient to solidify the mixture but a mass fraction of $1 \%$ was chosen to increase the strength of the gel. The gel was pumped into the shell after the EPS microspheres remained suspended in the gel and did not settle to the top anymore, but before the gel fully turned solid so it was still pumpable. An example of the thermal filling is shown in Figure 2. 


\subsection{Temperature measurements}

Inside the thin shell body, two wireless, self-powered data loggers with a built-in sensor were integrated, namely iButtons ${ }^{\circledast}$ (Maxim Integrated Products, California, USA). iButtons ${ }^{\circledast}$ are small, cylindrical chip-based electronic tags. Their internal battery is not rechargeable or replaceable so they are used until it is consumed. iButtons ${ }^{\circledR}$ have specific advantages essential for the fruit simulator (Maxim Integrated, 2013):

- wireless, stand-alone sensor/logger unit

- $\quad$ small (diameter $17 \mathrm{~mm}$, thickness $6 \mathrm{~mm}$ )

- a long autonomy, up to several years, depending on logging intervals

- available logging intervals are relevant to cold chain applications

- robust, durable stainless steel casing

- easy to program and download which does not require expert knowledge.

The installed sensor types depend on the temperature range of interest. In this study, we used iButton ${ }^{\circledR}$ Thermochron DS1922L-F5 (Maxim Integrated Products, USA, 8kB memory @ 8bit, resolution $0.0625^{\circ} \mathrm{C}$, accuracy $\pm 0.5^{\circ} \mathrm{C}$, from $-40{ }^{\circ} \mathrm{C}$ to $+85^{\circ} \mathrm{C}$, sampling rate from $1 \mathrm{~s}$ up to $273 \mathrm{~h}$ ). In the experiments, a measurement interval of $60 \mathrm{~s}$ was taken. The sensor at the surface was counter sunken in the artificial shell to not disturb the airflow around the fruit.

\subsection{Evaluating the performance of the artificial fruit}

\subsubsection{Filling}

The thermal conductivity of the water-carbohydrate-air gel mixture was measured and compared to the calculated values specified in Table 2. The thermal properties of three gel samples were determined using a guarded hot plate apparatus, which was constructed in-house. The apparatus satisfied the requirements of standard DIN 52612:1984 (withdrawn; replaced by DIN EN 12667). The samples (200 mm x $200 \mathrm{~mm} \times 20 \mathrm{~mm}$ thick) were placed between the hot upper plate $\left(30^{\circ} \mathrm{C} \pm 0.05^{\circ} \mathrm{C}\right)$ and the cold lower plate $\left(20^{\circ} \mathrm{C} \pm 0.05^{\circ} \mathrm{C}\right)$. Approximately $2 \mathrm{kPa}$ of pressure was applied to the gel during testing. The power required to maintain the defined temperature gradient (steady state) was measured allowing the thermal conductivity of the samples to be determined. The relative measurement uncertainty for the thermal conductivity was $2 \%$.

To evaluate the internal distribution of the filling in the shell, an X-ray computed tomography was performed. Radiographs were made by rotating the object in discrete steps of 0.25 degrees over an angle of 360 degrees. By reconstruction of the $2 \mathrm{D}$ radiographic images taken under different view angles using an $\mathrm{X}$-ray source and detector, a 3D image of the artificial fruit was obtained. The different components could be identified, based on differences in attenuation properties of the materials. The X-ray CT scans were made on a microCT setup consisting of a Viscom XT9160 TXD X-ray tube, a Micos UPR-160 F AIR high precision rotation table, and a Perkin Elmer XRD-1621-CN3ES X-ray detector. The scans were performed at $150 \mathrm{kV}$, with a field of view of $8.5 \times 8.5 \mathrm{~cm}^{2}$ and a pixel resolution of $48 \mu \mathrm{m}$. The images were segmented for further analysis using the commercial software Avizo Fire (Edition 9.2, FEl, Oregon, USA). 


\subsubsection{Cooling experiments on artificial fruit versus real fruit}

To evaluate the performance of the artificial fruit in mimicking the thermal response of real fruit, cooling experiments were performed in a climatic chamber (Feutron Klimasimulation GmbH, Feutron KPK 800). This climatic chamber has inner dimensions of $770 \times 1380 \times 745 \mathrm{~mm}$ so an inner volume of $800 \mathrm{~L}$. Air is supplied from the bottom via a perforated plate and is extracted on top of the chamber. The temperature range is -40 to $100{ }^{\circ} \mathrm{C}$ $+/-0.2{ }^{\circ} \mathrm{C}$, the relative humidity range is $10-95 \%+/-3 \%$ and the air speed is about $0.3 \mathrm{~m} \mathrm{~s}^{-1}$. This air speed leads to Biot numbers of around one, which means conductive heat transfer in the fruit and convective heat exchange with the air both will play a role in the cooling kinetics.

Apple fruit (cv. Braeburn) were purchased at a local retailer. The individual fruit was selected to have a weight that was as close as possible to that of the artificial fruit (Table 3). The artificial fruit and 10 real fruit were each equipped with an iButton ${ }^{\circledast}$ temperature sensor in the core. In the artificial fruit, a sensor was also placed in the enclosure at its surface (see Figure 1). The temperature sensor was inserted in the apples by cutting them in half, placing the sensor in the core and joining the two halves back together. This was done using tape, which sealed the entire cut plane. Prior to the experiment, all fruit were stabilized in another climatic room to $20^{\circ} \mathrm{C}$. Afterwards, the fruit were placed in the climatic chamber, which ran at $3^{\circ} \mathrm{C}$, and a cooling period of 225 min was monitored.

From the fruit core temperature profiles $\left(T_{c}\right)$, the fractional unaccomplished temperature change $(Y)$ was determined:

$$
Y=\frac{T_{c}-T_{a}}{T_{c, i}-T_{a}}
$$

where subscripts $c, i$ and $a$ represent the initial (core) temperature of each individual fruit (around $20^{\circ} \mathrm{C}$ ) and the set cooling air temperature of the climatic chamber $\left(3^{\circ} \mathrm{C}\right)$, respectively. From this $Y$ value, the half cooling time $\left(\mathrm{HCT}, t_{1 / 2}\right)$ and seven-eighths cooling times (SECT, $\left.t_{7 / 8}\right)$ were determined, which are the times required to reduce the temperature difference between the fruit and the cooling air by half $(Y=0.5)$ and seven eighths $(Y=0.125)$, respectively. The SECT is frequently used in commercial (pre)cooling operations because the fruit temperature at that value is acceptably close to the required storage temperature (Brosnan and Sun, 2001). These cooling times also enable a more straightforward and correct comparison of fruit cooling rates since the different fruit were not exactly at the same initial temperature. The reason is that the critical temperature to reach the SECT or HCT depends on this initial temperature $T_{c, i}$. The standard deviation on the initial fruit temperature between the 10 fruit was however small, namely below $0.1^{\circ} \mathrm{C}$.

\subsubsection{Cooling experiments on fruit simulators versus water-filled simulators}

In previous studies, water-filled fruit simulators have been used as substitutes for real fruit (section 2.1). The thermal properties of water, however, do not match those of real fruit (Table 2) since the latter also contains carbohydrates and air. As a result, an apple and its artificial fruit counterpart both float in water, whereas a water-filled fruit simulator will not. To investigate the errors induced by using water-filled spheres on the thermal 
response, two additional spherical fruit simulators were constructed (diameter $60 \mathrm{~mm}$, volume $0.113 \mathrm{~L}$ ), one filled with the water-carbohydrate-air mixture, corresponding to apple fruit (Table 2), and one filled with water. In both cases, a gelling agent (agar, mass fraction $1 \%$ ) was added to immobilize the liquid. Similar cooling experiments as described above (section 2.4.2) were performed. 


\section{Results}

\subsection{Manufactured artificial fruit}

Measurements were performed as first indicators for the performance of the manufactured artificial fruit, prior to the actual cooling experiments. First, the thermal conductivity of the filling was measured (section 2.4.1). An average value of $0.491 \mathrm{~W} \mathrm{~m}^{-1} \mathrm{~K}^{-1}$ (+/- 0.010 standard deviation) was obtained. There is a difference with the theoretical target value of $0.454 \mathrm{~W} \mathrm{~m}^{-1} \mathrm{~K}^{-1}$ (Table 2), which is lower. This difference is attributed to following reasons: (1) the thermal properties of the components of the mixture were taken from tabulated data (Table 2), but some differences with the actual used materials could be present; (2) the WCA gel mixture was produced manually in the lab in rather small quantities, by which small differences in composition were inherently present, but industrial manufacturing in larger volumes will reduce this effect; (3) the size of the EPS microspheres (0.5-1.5 $\mathrm{mm}$ ) is larger than that of the air pores in apple fruit ( $0.1 \mathrm{~mm}$, (Herremans et al., 2015)). The use of smaller particles could alleviate this. The test parameters used (absolute temperature, temperature gradient, pressure, etc.) and slight inconsistencies in the sample surfaces and thickness may also contribute in part for this discrepancy. Although a slightly higher thermal conductivity is obtained for the filling than expected, the obtained values are close (below $8 \%$ ). Also note that different models exist to estimate the thermal conductivity (Gulati and Datta, 2013), which can lead to slightly different predictions.

It should be noted that the shell, which has a lower thermal conductivity than apple fruit, and the presence of the iButtons ${ }^{\circledR}$ will also affect the thermal response of the fruit. However, achieving a similar thermal response of the composed artificial fruit, as compared to real fruit, is actually most essential (section 2.2). A certain degree of thermal mismatching was even argued to be acceptable (Redding et al., 2016).

Second, the density of the entire artificial fruit after filling was also determined based on the volume and its weight (including magnetic inlays and iButton sensors), i.e. $914 \mathrm{~kg} \mathrm{~m}^{-3}$. This value is very close to that of a real apple fruit (922 $\mathrm{kg} \mathrm{m}^{-3}$, Table 2).

Third, X-ray imaging is used to get insight of the artificial fruit after manufacturing to investigate the internal composition. The internal distribution of the filling inside the artificial fruit is shown in Figure 3. From such imaging, we identified that a small amount of entrapped air bubbles were entrained inside during filling. However, their amount was lower than $0.3 \%$ of the total volume of the fruit. The formation of such air voids during filling are a common problem (Redding et al., 2016). We also quantified the volume of EPS micro-spheres in the fruit, which led to a porosity of $15 \%$, which was only $2 \%$ different from the targeted porosity (13\%). This value is close and also well within the spread found for different individual fruit (Herremans et al., 2015). Note that no clear air gap between the two halves of the sphere was present. This good contact ensures that there is no thermal resistance between the halves and also avoids air penetration between the halves.

The effect of microbiological activity in the WCA gel over time was also evaluated by identifying if separate WCA gel samples were affected. No degradation could be visually observed over a period of several months. Note that when larger mass fractions of agar were used (e.g. 4 \%) some microbiological activity (indicated by a color change) could be observed over time, which was enhanced by exposure to light and higher temperatures. 



\subsection{Cooling experiments of fruit simulators versus real fruit}

First, the repeatability of the experiments in the climatic chamber was evaluated and was found to be very good: the differences in half-cooling time (HCT) and seven-eighths cooling time (SECT) for a single artificial fruit used in different cooling experiments were less than $0.1 \%$. Different artificial fruit were also produced to check the repeatability in production of the artificial fruit, particularly regarding to its filling. Differences in HCT and SECT between 3 artificial fruit were about $5 \%$. These differences are attributed to small differences in production of the thermal filling, as production was done on a small scale where each fruit was produced at a different point in time. Such differences will become less if larger volumes of filling are produced on an industrial scale in order to manufacture large numbers of artificial fruit.

In Figure 4, the fruit cooling rates of the artificial fruit and the 10 apple fruits are evaluated by means of their core temperatures as a function of time. The air temperature and the temperature of the sensor located at the artificial fruit surface (see Figure 1) are also shown. The HCT and SECTs extracted from these profiles are represented in Table 3.

From these data it is clear that the artificial fruit responds thermally very similar to real apple fruit. The temperature at the artificial fruit surface decreases directly after imposing the temperature drop, but the core temperature clearly shows a lag due to the thermal capacity. The obtained values for HCT ( $1 \mathrm{~h}$ ) and SECT ( $2 \mathrm{~h}$ ) are in the same range as previously reported values for fruit packed in ventilated packaging (Berry et al., 2017; Defraeye et al., 2014). However, our values correspond rather to the HCT and SECT in the high airspeed range of previous studies. The reason is that single fruit were convectively cooled instead of packed produce. In the latter case, the monitored fruit would be more sheltered by the packaging and the other fruit. 


\subsection{Cooling of artificial fruit versus water-filled simulators}

The cooling behavior of a spherical artificial fruit, filled with the aforementioned water-carbohydrate-air (WCA) gel mixture, and a shell filled with water-based gel are compared. In Figure 5, the core temperature of both simulators is shown as a function of time. The air temperature and the temperature of the sensors located at the simulator surfaces are also shown. The HCT and SECTs from these core temperature profiles are represented in Table 4.

There is a clear difference between the convective cooling of the fruit with the different fillings, where a waterbased gel cools slower, due to the different thermal properties (see Table 2). This slower convective cooling behavior for the water-filled simulator was cross-checked and confirmed by finite element simulations (results not reported). The differences were also noticeable in the surface temperature sensor data. This clearly indicates that the WCA filling should be preferred over just using water, even in a gel form. This WCA filling was based on the real apple fruit composition, using a biomimetic approach, and matched the thermal response of apple fruit well (Figure 4). 


\section{Discussion}

\subsection{Current limitations}

Despite its many advantages compared to present temperature measurement practices or fruit simulators, there are some restrictions to the artificial fruit. As with several other sensors, the artificial fruit sensor has to be manually placed inside the packaging, and has to be retrieved afterwards for readout. Such installation is typically done during packaging and prior to loading. As such, the sensor can only be removed, so read out, after one or more unit operations (precooling, transport, storage) in the cold chain. Only a-posteriori, offline temperature history information is available, in contrast to the real-time data from some wireless RFID smart sensor tags.

The heat of respiration that is produced by fruit is not generated explicitly inside the artificial fruit. However, this heat of respiration for a single fruit is very low, e.g. about $15 \mathrm{~mW} /$ fruit for apple ((ASHRAE, 2010), $180 \mathrm{~g}$ apple fruit at $20^{\circ} \mathrm{C}$ ) or $30-70 \mathrm{~mW} /$ fruit for banana ((ASHRAE, 2010; Kader, 2017), $180 \mathrm{~g}$ ripe banana fruit at $20^{\circ} \mathrm{C}$ ). Therefore the impact on the core temperature for a single fruit can be considered negligible and below the sensor accuracy. This assumption was confirmed as well in literature (Redding et al., 2016). Nevertheless, the heat of respiration produced by the rest of the cargo can be picked up by the artificial fruit, by which hot spots can still effectively be identified. The latent heat due to evaporation of water from the fruit can be neglected for produce simulators, as confirmed by (Redding et al., 2016).

\subsection{Future developments}

A future development is to integrate a sensor/logger system in the fruit which enables wireless transmission to allow real-time monitoring. Another further development would be the inclusion of a humidity sensor at the fruit surface (e.g. iButton Hygrochron ${ }^{\circledR}$ ), which can be used to assess the risk of surface condensation and microbial activity at the fruit surface. Finally, the artificial fruits should be produced in larger quantities, such as on an industrial scale. When the filling is produced in larger batches, the uniformity of the filling will improve, compared to the current lab-scale production. This will reduce the thermal response differences between different artificial fruit even more.

\subsection{Application to other fruit}

The concept and performance of the artificial fruit was demonstrated for apple fruit (cv. Braeburn) in this study. The methodology can easily be applied for other fruit or apple cultivars as well, which is currently performed by the authors. Particularly the fact that fruit composition data is tabulated in literature for many types of fruit (e.g. (ASHRAE, 2010)), makes that new fillings can be easily designed for other fruit species of interest.

A particular advantage of the artificial fruit, or fruit simulators in general, is that each simulator reacts thermally similar. Fruit pulp temperature measurements in real fruit, on the other hand, are always dependent on the size and shape of the individual fruit that is monitored but also on the location and depth with which the sensor is inserted. Particularly for fruit with a complex fruit shape and features (for example mango with seed), this variability in fruit size and shape and sensor location can introduce random errors in the data, making them less reliable. The uniformity in size, shape and sensor location of the artificial fruit however provides a way to measure fruit pulp temperature in a very repeatable way. Such standardization is essential in the cold chain. 


\subsection{Applications and perspectives for commercial cold chains}

The artificial fruit is useful for many different cold-chain stakeholders. Manufacturers of refrigerated containers and refrigeration units need to demonstrate the feasibility of new cooling hardware or control software to their customers by means of field trails on commercial shipments. The artificial fruit can give them better means for such demonstration, which can provide them with a commercial advantage. Recently, Maersk Container Industries relied on over 200 field trails to demonstrate the efficacy of their new QUEST control software for refrigerated containers (Lukasse et al., 2011).

The artificial fruit could also be used to provide clarity in claims of wholesalers or importers towards the producers or exporters regarding non-satisfactory product quality, as the loggers can remain inside the packaging all the way up to the retailer. The artificial fruit can also supplement the very few temperature probes that are placed inside commercial shipments (e.g. 3 UDSA-approved point sensors per refrigerated container), as these do not always accurately reflect the cooling behavior of the entire cargo (Defraeye et al., 2016). In addition to intransport use, the large amount of precooling facilities, cold stores and ripening facilities handling fruit could use the artificial fruit to improve monitoring of the thermal history of their perishable goods. As such, surprises in quality status and incidence of spoilage upon arrival of the fruit at the end of the line, can be clarified.

Finally, in postharvest technology research, the use of the artificial fruit can save a significant amount of time in setting up both lab-scale and field experiments, and can avoid the use of wired hardware. In addition, it becomes easier to cover a large spatial resolution within the cargo and to perform long-term monitoring in field tests throughout the entire chain.

\section{Conclusion}

This study proposed a new kind of fruit simulator - an artificial fruit - which was engineered specifically to match the thermal response of real fruit as close as possible. Each of the components of the simulator was designed, and combined successfully into the artificial fruit. The artificial apple fruit was shown to react thermally very similar to real apple fruit. Compared to water-filled simulators, the thermal response of the artificial fruit was also shown to be different, but more accurate than water-filled simulators.

This artificial fruit enables to measure in a very repeatable manner the thermal history of fruit pulp throughout the entire cold chain. Its ease of use make it especially attractive for use in commercial cold chains and installation at multiple locations in the cargo. Thereby heterogeneities in cargo cooling, and associated quality issues, can be identified. The performance of the current artificial fruit can be improved in a next step by producing it at industrial scale, to reduce the cost, and by including more sensors or wireless data-transfer capabilities. 


\section{Acknowledgements}

We acknowledge the support of the Commission for Technology and Innovation (CTI, Switzerland, project nr.

18155.2 PFLS-LS), of the World Food System Center (WFSC) of ETH Zürich (www.worldfoodsystem.ethz.ch) and of the Flanders Innovation \& Entrepreneurship (VLAIO, project IWT-120745). 


\section{References}

Allais, I., Alvarez, G., Flick, D., 2006. Modelling cooling kinetics of a stack of spheres during mist chilling. J. Food Eng. 72, 197-209. doi:10.1016/j.jfoodeng.2004.11.010

Alvarez, G., Bournet, P.E., Flick, D., 2003. Two-dimensional simulation of turbulent flow and transfer through stacked spheres. Int. J. Heat Mass Transf. 46, 2459-2469. doi:10.1016/S0017-9310(02)00546-X

Ambaw, A., Bessemans, N., Gruyters, W., Gwanpua, S.G., Schenk, A., De Roeck, A., Delele, M.A., Verboven, P., Nicolai, B.M., 2016. Analysis of the spatiotemporal temperature fluctuations inside an apple cool store in response to energy use concerns. Int. J. Refrig. 66, 156-168. doi:10.1016/j.ijrefrig.2016.02.004

ASHRAE, 2010. ASHRAE Handbook - Refrigeration: systems and applications (SI edition). Atlanta.

Berry, T.M., Fadiji, T.S., Defraeye, T., Opara, U.L., 2017. The role of horticultural carton vent hole design on cooling efficiency and compression strength: A multi-parameter approach. Postharvest Biol. Technol. 124, 62-74. doi:10.1016/j.postharvbio.2016.10.005

Brosnan, T., Sun, D.W., 2001. Precooling techniques and applications for horticultural products - a review. Int. J. Refrig. 24, 154-170.

Choe, Y.C., Park, J., Chung, M., Moon, J., 2009. Effect of the food traceability system for building trust: Price premium and buying behavior. Inf. Syst. Front. 11, 167-179. doi:10.1007/s10796-008-9134-z

Chuntranuluck, S., Wells, C.M., Cleland, A.C., 1998. Prediction of chilling times of foods in situations where evaporative cooling is significant-Part 3. Applications. J. Food Eng. 37, 143-157. doi:10.1016/S0260$8774(98) 00089-2$

De Castro, L.R., Vigneault, C., Cortez, L.A.B., 2005. Cooling performance of horticultural produce in containers with peripheral openings. Postharvest Biol. Technol. 38, 254-261. doi:10.1016/j.postharvbio.2005.07.004

De Castro, L.R., Vigneault, C., Cortez, L.A.B., 2005. Effect of container openings and airflow rate on energy required for forced-air cooling of horticultural produce. Can. Biosyst. Eng. 47, 1-9.

De Castro, L.R., Vigneault, C., Cortez, L. a. B., 2004. Container opening design for horticultural produce cooling efficiency. Food, Agric. Environ. 2, 135-140.

Defraeye, T., Cronjé, P., Berry, T., Opara, U.L., East, A., Hertog, M., Verboven, P., Nicolai, B., 2015a. Towards integrated performance evaluation of future packaging for fresh produce in the cold chain. Trends Food Sci. Technol. 44, 201-225. doi:10.1016/j.tifs.2015.04.008

Defraeye, T., Lambrecht, R., Delele, M.A., Tsige, A.A., Opara, U.L., Cronjé, P., Verboven, P., Nicolai, B., 2014. Forced-convective cooling of citrus fruit: Cooling conditions and energy consumption in relation to package design. J. Food Eng. 121, 118-127. doi:10.1016/j.jfoodeng.2013.08.021

Defraeye, T., Lambrecht, R., Tsige, A.A., Delele, M.A., Opara, U.L., Cronjé, P., Verboven, P., Nicolai, B., 2013. Forced-convective cooling of citrus fruit: Package design. J. Food Eng. 118, 8-18. 
doi:10.1016/j.jfoodeng.2013.03.026

Defraeye, T., Nicolai, B., Kirkman, W., Moore, S., Niekerk, S. van, Verboven, P., Cronjé, P., 2016. Integral performance evaluation of the fresh-produce cold chain: A case study for ambient loading of citrus in refrigerated containers. Postharvest Biol. Technol. 112, 1-13. doi:10.1016/j.postharvbio.2015.09.033

Defraeye, T., Verboven, P., Opara, U.L., Nicolai, B., Cronjé, P., 2015b. Feasibility of ambient loading of citrus fruit into refrigerated containers for cooling during marine transport. Biosyst. Eng. 134, 20-30. doi:10.1016/j.biosystemseng.2015.03.012

Dehghannya, J., Ngadi, M., Vigneault, C., 2012. Transport phenomena modelling during produce cooling for optimal package design: Thermal sensitivity analysis. Biosyst. Eng. 111, 315-324. doi:10.1016/j.biosystemseng.2012.01.001

Delele, M.A., Ngcobo, M.E.K., Getahun, S.T., Chen, L., Mellmann, J., Opara, U.L., 2013a. Studying airflow and heat transfer characteristics of a horticultural produce packaging system using a 3-D CFD model. Part II: Effect of package design. Postharvest Biol. Technol. 86, 546-555. doi:10.1016/j.postharvbio.2013.08.015

Delele, M.A., Ngcobo, M.E.K., Getahun, S.T., Chen, L., Mellmann, J., Opara, U.L., 2013b. Studying airflow and heat transfer characteristics of a horticultural produce packaging system using a 3-D CFD model. Part I: Model development and validation. Postharvest Biol. Technol. 86, 536-545. doi:10.1016/j.postharvbio.2013.08.014

do Nascimento Nunes, M.C., Nicometo, M., Emond, J.P., Melis, R.B., Uysal, I., 2014. Improvement in fresh fruit and vegetable logistics quality: berry logistics field studies. Philos. Trans. R. Soc. A Math. Phys. Eng. Sci. 372, 20130307-20130307. doi:10.1098/rsta.2013.0307

East, A.R., 2011. Accelerated libraries to inform batch sale scheduling and reduce postharvest losses of seasonal fresh produce. Biosyst. Eng. 109, 1-9. doi:10.1016/j.biosystemseng.2011.01.008

EU-Catrene, 2012. EU-Catrene project Pasteur: Perishables Monitoring through Smart Tracking of Lifetime and Quality by RFID (www.aeneas-office.eu) [WWW Document]. URL http://www.aeneasoffice.eu/web/downloads/ct204-pasteur-project_profile-final_7-6-11.pdf

Ferrua, M.J., Singh, R.P., 2009. Design guidelines for the forced-air cooling process of strawberries. Int. J. Refrig. 32, 1932-1943. doi:10.1016/j.jirefrig.2009.07.011

Gruyters, W., Defraeye, T., Berry, T., Ambaw, A., Opara U.L, Verboven, P., Nicolai, B., 2017. Precooling of “Granny Smith" apples: trade-offs between cooling rate and energy consumption. Submitted.

Gulati, T., Datta, A.K., 2013. Enabling computer-aided food process engineering: Property estimation equations for transport phenomena-based models. J. Food Eng. 116, 483-504. doi:10.1016/j.jfoodeng.2012.12.016

Gustavsson, J., Cederberg, C., Sonesson, U., van Otterdijk, R., Meybeck, A., 2011. Global food losses and food waste: Extend, causes and prevention. Rome, Italy.

Herremans, E., Verboven, P., Bongaers, E., Estrade, P., Verlinden, B.E., Wevers, M., Hertog, M.L.A.T.M., Nicolai, B.M., 2013. Characterisation of "Braeburn" browning disorder by means of X-ray micro-CT. Postharvest Biol. 
Technol. 75, 114-124. doi:10.1016/j.postharvbio.2012.08.008

Herremans, E., Verboven, P., Verlinden, B.E., Cantre, D., Abera, M., Wevers, M., Nicolaï, B.M., 2015. Automatic analysis of the 3-D microstructure of fruit parenchyma tissue using X-ray micro-CT explains differences in aeration. BMC Plant Biol. 15, 264. doi:10.1186/s12870-015-0650-y

Hertog, M.L.A.T.M., Uysal, I., Verlinden, B.M., Nicolaï, B.M., 2014. Shelf life modelling for first-expired-first-out warehouse management. Philos. Trans. R. Soc. 372, 15. doi:10.1098/rsta.2013.0306

Hoang, M.H., Laguerre, O., Moureh, J., Flick, D., 2012. Heat transfer modelling in a ventilated cavity loaded with food product: Application to a refrigerated vehicle. J. Food Eng. 113, 389-398. doi:10.1016/j.jfoodeng.2012.06.020

Jedermann, R., Geyer, M., Praeger, U., Lang, W., 2013. Sea transport of bananas in containers - Parameter identification for a temperature model. J. Food Eng. 115, 330-338. doi:10.1016/j.jfoodeng.2012.10.039

Jedermann, R., Nicometo, M., Uysal, I., Lang, W., 2014a. Reducing food losses by intelligent food logistics. Philos. Trans. A. Math. Phys. Eng. Sci. 372, 20130302. doi:10.1098/rsta.2013.0302

Jedermann, R., Potsch, T., Lloyd, C., 2014b. Communication techniques and challenges for wireless food quality monitoring. Philos. Trans. R. Soc. A Math. Phys. Eng. Sci. 372, 20130304-20130304. doi:10.1098/rsta.2013.0304

Jedermann, R., Praeger, U., Geyer, M., Lang, W., 2014c. Remote quality monitoring in the banana chain. Philos. Trans. A. Math. Phys. Eng. Sci. 372, 20130303. doi:10.1098/rsta.2013.0303

Jimenez-Ariza, T., Correa, E.C., Diezma, B., Silveira, A.C., Zocalo, P., Arranz, F.J., Moya-Gonzalez, A., Garrido-Izard, M., Barreiro, P., Ruiz-Altisent, M., 2014. The Phase Space as a New Representation of the Dynamical Behaviour of Temperature and Enthalpy in a Reefer monitored with a Multidistributed Sensors Network. Food Bioprocess Technol. 7, 1793-1806. doi:10.1007/s11947-013-1191-8

Kader, A., 2017. Banana - Recommendations for maintaining postharvest quality [WWW Document]. UC Davis Commod. fact sheets. URL http://postharvest.ucdavis.edu/Commodity_Resources/Fact_Sheets/Datastores/Fruit_English/?uid=9\&ds=7 98

Laniel, M., Émond, J.P., 2010. Mapping of RFID tag readability in relation to the food content in a refrigerated sea container at 915 MHz. Innov. Food Sci. Emerg. Technol. 11, 703-706. doi:10.1016/j.ifset.2010.06.005

Laniel, M., Émond, J.P., Altunbas, A.E., 2011. Effects of antenna position on readability of RFID tags in a refrigerated sea container of frozen bread at 433 and $915 \mathrm{MHz}$. Transp. Res. Part C Emerg. Technol. 19, 1071-1077. doi:10.1016/j.trc.2011.06.008

Lu, J., Dev, S.R.S., Vijaya Raghavan, G.S., Vigneault, C., 2009. Simulation of a forced-air-twin-chamber for measuring heat treatment uniformity in harvested tomatoes. J. Food Eng. 95, 636-647.

doi:10.1016/j.jfoodeng.2009.06.031 
Lukasse, L.J.S., Baerentz, M.B., Kramer-Cuppen, J.E.D., 2011. Quest II : Reduction of CO2 emissions of reefer containers, in: 23rd IIR International Congress of Refrigeration. Prague, pp. 3203-3210.

Lütjen, M., Dittmer, P., Veigt, M., 2013. Quality driven distribution of intelligent containers in cold chain logistics networks. Prod. Eng. 7, 291-297. doi:10.1007/s11740-012-0433-3

Martínez-Monzó, J., Barat, J.M., González-Martínez, C., Chiralt, A., Fito, P., 2000. Changes in thermal properties of apple due to vacuum impregnation. J. Food Eng. 43, 213-218. doi:10.1016/S0260-8774(99)00152-1

Maxim Integrated, 2013. iButton Temperature Loggers with 8KB Data-Log Memory.

O’Sullivan, J., Ferrua, M.J., Love, R., Verboven, P., Nicolai, B., East, A., 2016. Modelling the forced-air cooling mechanisms and performance of polylined horticultural produce. Postharvest Biol. Technol. 120, 23-35. doi:10.1016/j.postharvbio.2016.05.008

Olatunji, J.R., Love, R.J., Shim, Y.M., Ferrua, M.J., East, A.R., 2017. Quantifying and visualising variation in batch operations: A new heterogeneity index. J. Food Eng. 196, 81-93. doi:10.1016/j.jfoodeng.2016.10.004

Pang, Z., Chen, Q., Han, W., Zheng, L., 2015. Value-centric design of the internet-of-things solution for food supply chain: Value creation, sensor portfolio and information fusion. Inf. Syst. Front. 17, 289-319. doi:10.1007/s10796-012-9374-9

PPECB, 2016a. PPECB procedural manual on general procedure, loading and carrying temperature requirements for the export of perishable products. HP34C/PP04.03-11: Procedure for in -transit cold treatment of specified quarantine pest in citrus shipped from South Africa.

PPECB, 2016b. PPECB procedural manual on general procedure, loading and carrying temperature requirements for the export of perishable products. PPO4.02-21F02: check list for cold store and ambient loading facilities registration.

Redding, G.P., Yang, A., Shim, Y.M., Olatunji, J., East, A., 2016. A review of the use and design of produce simulators for horticultural forced-air cooling studies. J. Food Eng. 190, 80-93. doi:10.1016/j.jfoodeng.2016.06.014

Rogge, S., Beyene, S.D., Herremans, E., Hertog, M.L., Defraeye, T., Verboven, P., Nicolai, B.M., 2014. A geometrical model generator for quasi-axisymmetric biological products. Food Bioprocess Technol. 7, 1783-1792. doi:10.1007/s11947-013-1169-6

Rogge, S., Defraeye, T., Herremans, E., Verboven, P., Nicolaï, B.M., 2015. A 3D contour based geometrical model generator for complex-shaped horticultural products. J. Food Eng. 157, 24-32. doi:10.1016/j.jfoodeng.2015.02.006

Swedberg, C., 2011. Hartford encourages its food-industry clients to deploy RFID temperature tags. RFID J. 1-3.

Thompson, 2008. Commercial cooling of fruits, vegetables and flowers, University of California. University of California, California. 
Thompson, J.F., 2004. Pre-cooling and storage facilities, in: USDA (Ed.), USDA Agriculture Handbook Number 66: The Commercial Storage of Fruits, Vegetables, and Florist and Nursery Stocks. USDA, pp. 1-10.

USDA-APHIS, 2017. USDA Treatment manual 10/2016-01. [WWW Document]. URL https://www.aphis.usda.gov/import_export/plants/manuals/ports/downloads/treatment.pdf

Vigneault, C., de Castro, L.R., 2005. Produce-simulator property evaluation for indirect airflow distribution measurement through horticultural crop package. J. Food, Agric. Environ. 3, 67-72.

Vigneault, C., Goyette, B., de Castro, L.R., 2006. Maximum slat width for cooling efficiency of horticultural produce in wooden crates. Postharvest Biol. Technol. 40, 308-313. doi:10.1016/j.postharvbio.2006.01.005

Wu, W., Häller, P., Cronjé, P., Defraeye, T., 2017. Full-scale experiments in 40-pallet forced-air precoolers for citrus fruit: Impact of packaging type and fruit size on cooling rate and heterogeneity. Submitted.

Zou, Z., Chen, Q., Uysal, I., Zheng, L., 2014. Radio frequency identification enabled wireless sensing for intelligent food logistics. Philos. Trans. R. Soc. A 372, 20130313. doi:10.1098/rsta.2013.0313 


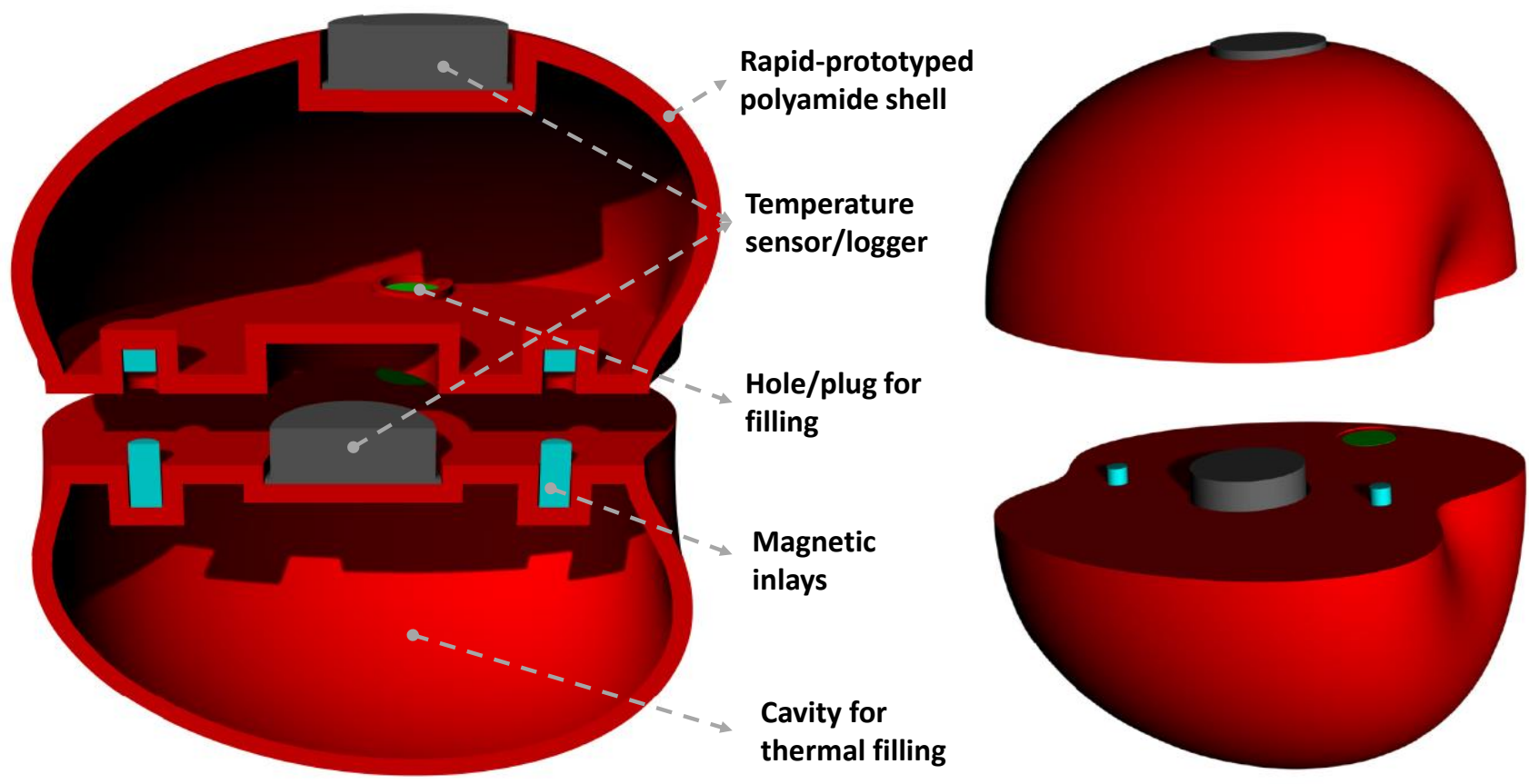

Figure 1. Illustration of the artificial apple fruit and its various components. 

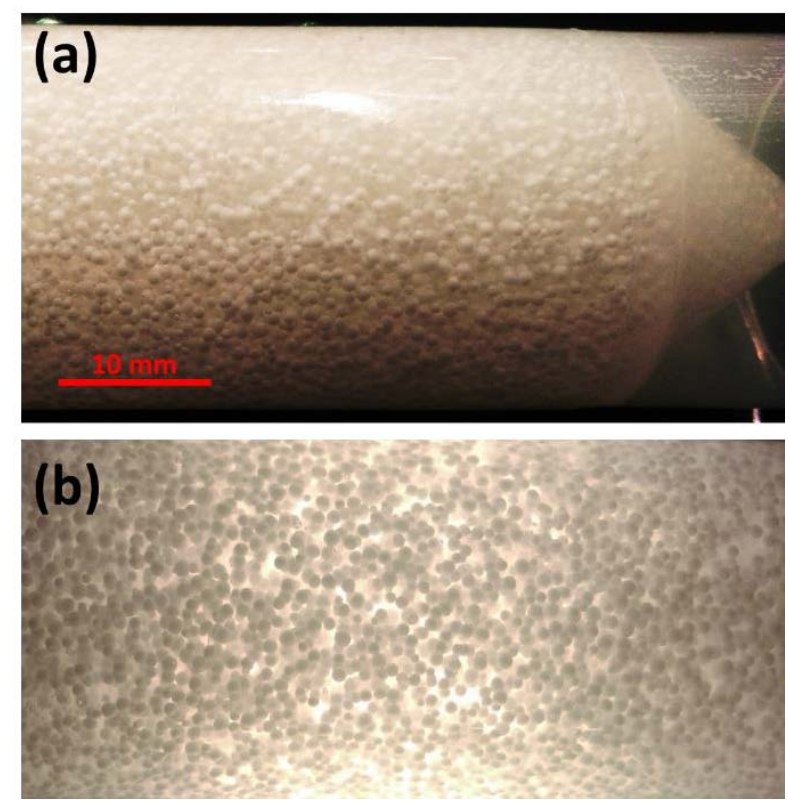

Figure 2. Pictures of water-carbohydrate-air gel filling: (a) in plastic container, (b) in plastic container with backlighting (diameter EPS spheres 0.5-1.5 mm). 

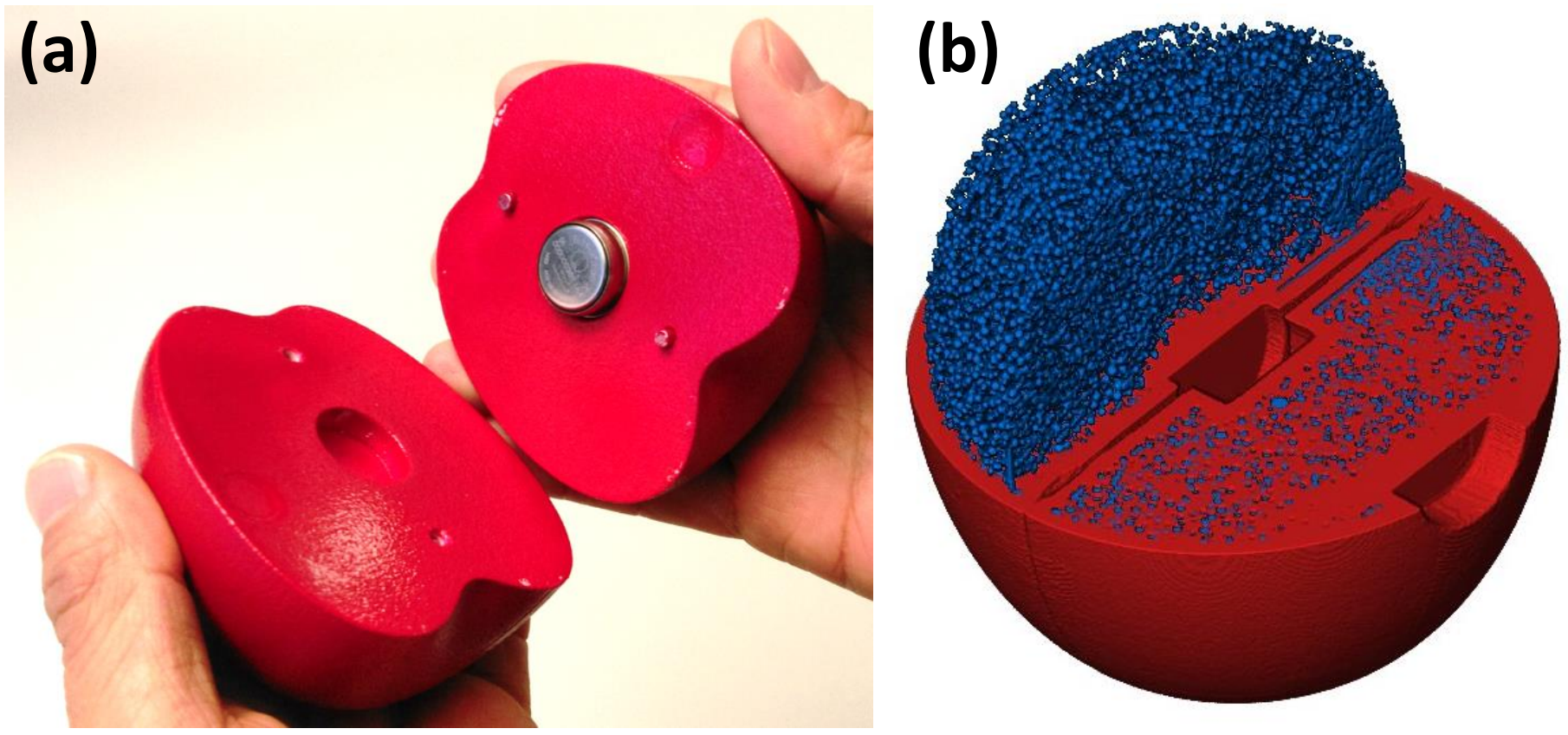

Figure 3. Artificial fruit: (a) picture, (b) cross-section, imaged with X-ray computed tomography, where the EPS particles are segmented out in blue and the shell and the filling in red. 


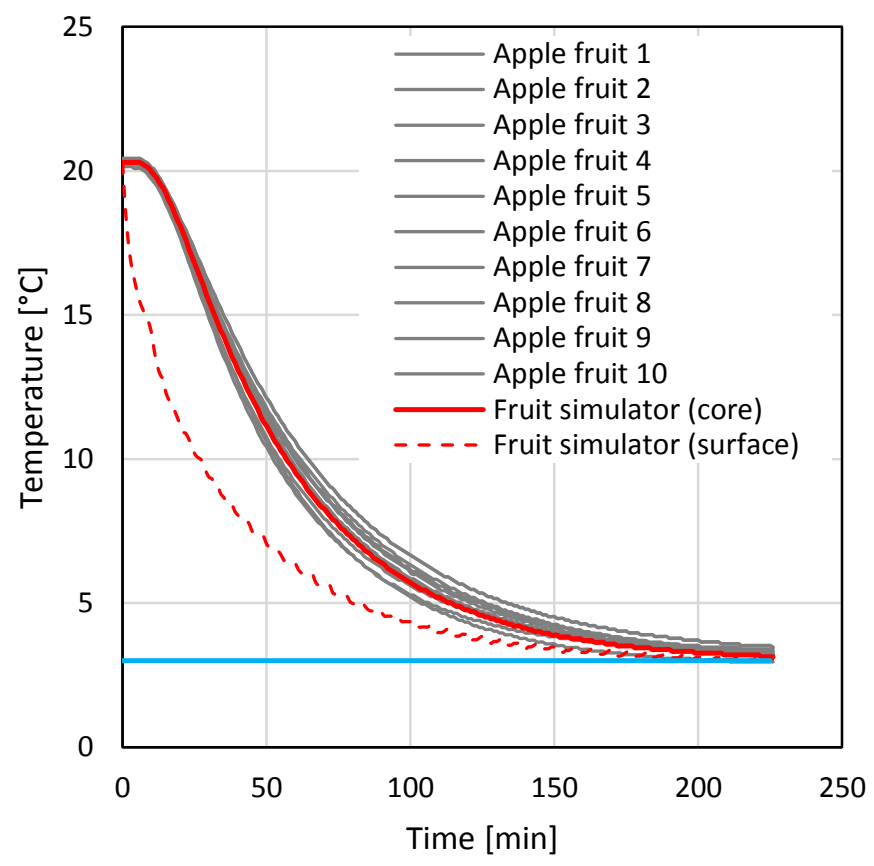

Figure 4. Core temperature of the artificial fruit simulator and 10 apple fruits as a function of time. The temperature at the surface of the fruit simulator (dashed red line) and set air temperature of the climatic chamber (blue line) are also shown. 


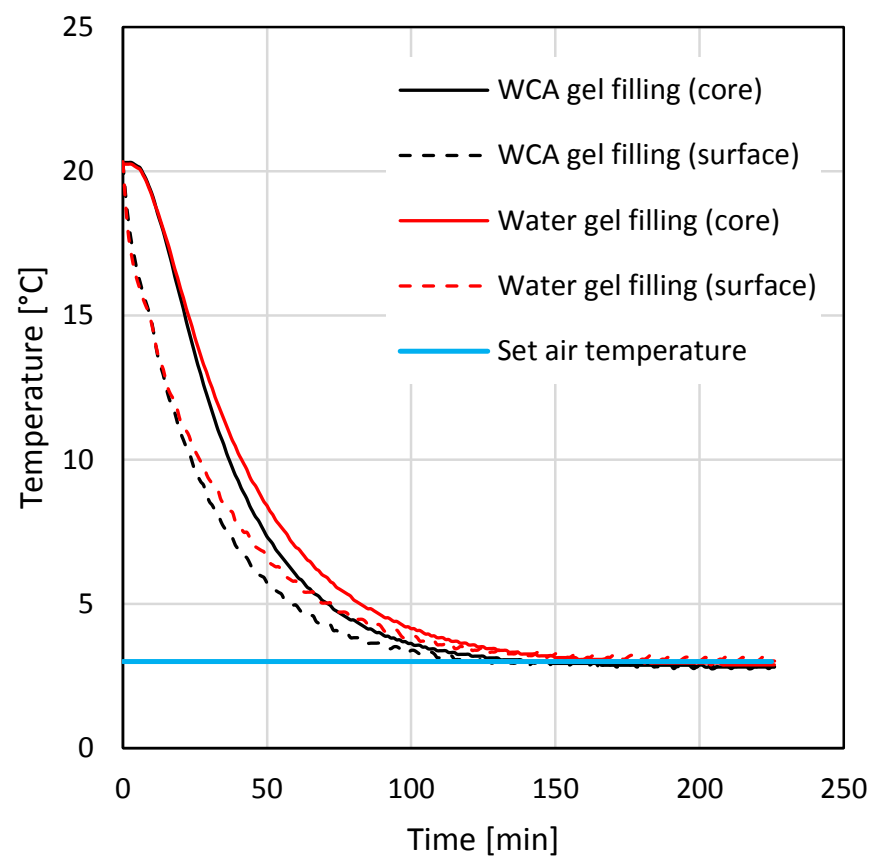

Figure 5. Temperature of spherical shells, filled with a WCA mixture (same as artificial fruit) and with a water gel, as a function of time. Both core and surface temperature of these produce simulators are given as well as the set air temperature in the climatic chamber. 
Tables

Table 1. Previous studies on fruit simulators.

\begin{tabular}{|c|c|c|c|c|c|c|c|}
\hline Source & Material & $\begin{array}{l}\text { Density } \\
{\left[\mathrm{kg} \mathrm{m}^{-3}\right]}\end{array}$ & $\begin{array}{l}\text { Specific heat } \\
\text { capacity } \\
{\left[\mathrm{J} \mathrm{kg}^{-1} \mathrm{~K}^{-1}\right]}\end{array}$ & $\begin{array}{l}\text { Thermal } \\
\text { conductivity } \\
{\left[\mathrm{W} \mathrm{m}^{-1} \mathrm{~K}^{-1}\right]}\end{array}$ & $\begin{array}{l}\text { Thermal } \\
\text { diffusivity }\left(\times 10^{6}\right) \\
{\left[\mathrm{m}^{2} \mathrm{~s}^{-1}\right]}\end{array}$ & $\begin{array}{l}\text { Characteristic } \\
\text { length } \\
{[\mathrm{mm}]}\end{array}$ & $\begin{array}{l}\text { Air speed } \\
{\left[\mathrm{m} \mathrm{s}^{-1}\right]}\end{array}$ \\
\hline (Allais et al., 2006) & $\begin{array}{l}\text { Celluloid gel-filled } \\
\text { sphere }\end{array}$ & 1013 & 4100 & 0.519 & 0.12 & 38 & $0.35-2.8$ \\
\hline $\begin{array}{l}\text { (Vigneault and de Castro, } \\
\text { 2005) }\end{array}$ & Polymer sphere & 1670 & 1112 & 0.684 & 0.37 & 52 & $0.065-1.05$ \\
\hline (Vigneault et al., 2006) & Polymer sphere & 1670 & 1112 & 0.684 & 0.37 & 52 & $0.1-2.5$ \\
\hline (Dehghannya et al., 2012) & Polymer sphere & 1670 & 1112 & 0.684 & 0.37 & 52 & 0.51 \\
\hline $\begin{array}{l}\text { (Larissa R. De Castro et al., } \\
\text { 2005) }\end{array}$ & Polymer sphere & 1670 & 1112 & 0.684 & 0.37 & 52 & $0.03-1.04$ \\
\hline (Delele et al., 2013b) & $\begin{array}{l}\text { Water-filled plastic } \\
\text { ball }\end{array}$ & 1033 & 3859 & 0.467 & 0.12 & 80 & $0.03-0.13$ \\
\hline $\begin{array}{l}\text { (Chuntranuluck et al., } \\
\text { 1998) }\end{array}$ & $\begin{array}{l}\text { Aluminium cylindrical } \\
\text { tube filled with Tylose }\end{array}$ & - & - & 0.5 & 0.13 & $72-142$ & $0.5-3.4$ \\
\hline
\end{tabular}


Table 2. Thermal properties of apple fruit and of artificial fruit components.

\begin{tabular}{|c|c|c|c|c|c|c|}
\hline & $\begin{array}{l}\text { Mass } \\
\text { fraction [\%] }\end{array}$ & $\begin{array}{l}\text { Density } \\
{\left[\mathrm{kg} \mathrm{m}^{-3}\right]}\end{array}$ & $\begin{array}{l}\text { Specific heat } \\
\text { capacity } \\
{\left[\mathrm{J} \mathrm{kg}^{-1} \mathrm{~K}^{-1}\right]}\end{array}$ & $\begin{array}{l}\text { Thermal conductivity } \\
{\left[\mathrm{W} \mathrm{m}^{-1} \mathrm{~K}^{-1}\right]}\end{array}$ & $\begin{array}{l}\text { Thermal } \\
\text { diffusivity (x } \\
\left.10^{6}\right) \\
{\left[\mathrm{m}^{2} \mathrm{~s}^{-1}\right]}\end{array}$ & Source \\
\hline Water & 83.9 & 996 & 4129 & 0.604 & 0.15 & (ASHRAE, 2010) \\
\hline Carbohydrates & 15.3 & 1593 & 1586 & 0.227 & 0.09 & (ASHRAE, 2010) \\
\hline Air & $\begin{array}{l}0 \text { (but } \\
\text { porosity = } \\
13 \%)\end{array}$ & 1.225 & 1006 & 0.0242 & 19.6 & (Defraeye, 2011) \\
\hline Apple fruit & - & 922 & 3722 & 0.451 & 0.13 & $\begin{array}{l}\text { Calculated based } \\
\text { on composition } \\
\text { from (ASHRAE, } \\
\text { 2010) }\end{array}$ \\
\hline \multicolumn{7}{|l|}{ Filling of artificial fruit components } \\
\hline Water & 83.9 & 996 & 4129 & 0.604 & 0.15 & (ASHRAE, 2010) \\
\hline Carbohydrates (sucrose) & 15.1 & 1593 & 1586 & 0.227 & 0.09 & (ASHRAE, 2010) \\
\hline Gelling agent (agar) & 1 & 1593 & 1586 & 0.227 & 0.09 & (ASHRAE, 2010) \\
\hline $\begin{array}{l}\text { EPS microspheres (air substitute - } \\
\text { volume fraction } 13 \% \text { ) }\end{array}$ & 0 & 15 & 1500 & 0.0380 & 1.69 & (Hens, 2003) \\
\hline Artificial fruit (target values) & - & 925 & 3721 & 0.454 & 0.13 & $\begin{array}{l}\text { Calculated based } \\
\text { on composition } \\
\text { from (ASHRAE, } \\
\text { 2010) }\end{array}$ \\
\hline
\end{tabular}


Table 3. Weight, half cooling time and seven-eighths cooling time from the fruit core temperature in the 10 apple fruit (including the average value) and the artificial fruit simulator, as well as the difference between both.

\begin{tabular}{|c|c|c|c|}
\hline & Weight [g] & HCT [min] & SECT [min] \\
\hline Apple fruit 1 & 189 & 48 & 108 \\
\hline Apple fruit 2 & 185 & 47 & 108 \\
\hline Apple fruit 3 & 182 & 52 & 123 \\
\hline Apple fruit 4 & 178 & 51 & 121 \\
\hline Apple fruit 5 & 173 & 49 & 115 \\
\hline Apple fruit 6 & 188 & 54 & 130 \\
\hline Apple fruit 7 & 182 & 54 & 130 \\
\hline Apple fruit 8 & 187 & 51 & 121 \\
\hline Apple fruit 9 & 181 & 47 & 111 \\
\hline Apple fruit 10 & 183 & 44 & 102 \\
\hline Apple fruit average & 183 & 49 & 117 \\
\hline Apple fruit standard deviation & 5 & 3 & 10 \\
\hline Artificial fruit (with sensor) & 184 & 48 & 111 \\
\hline DIFFERENCE & & $3 \%$ & $5 \%$ \\
\hline
\end{tabular}

Table 4. Half cooling time and seven-eighths cooling time from the fruit core temperature in the spherical fruit simulators with the WCA gel mixture (as the artificial fruit) and the water-based gel filling, as well as the difference between both.

\begin{tabular}{|l|r|r|}
\hline & HCT & \multicolumn{1}{l|}{ SECT } \\
\hline WCA gel mixture & 32 & 69 \\
\hline Water gel filling & 35 & 80 \\
\hline DIFFERENCE & $10 \%$ & $16 \%$ \\
\hline
\end{tabular}

